\title{
Comparison between the effect of epidural anesthesia combined with epidural analgesia and general anesthesia combined with intravenous analgesia on prognosis of ovarian cancer patients
}

\author{
SHUIGEN ZHONG ${ }^{1}$, XIAOXUE ZHONG $^{2}$, XIAOMEI ZHONG ${ }^{3}$ and YANLONG LIU ${ }^{1}$ \\ ${ }^{1}$ Department of Anesthesiology, Jiangxi Provincial People's Hospital; ${ }^{2}$ Department of Nephrology, \\ Jiangxi Hospital of Traditional Chinese Medicine; ${ }^{3}$ Department of Ophtalmology, \\ The First Hospital of Nanchang, Nanchang, Jiangxi 330006, P.R. China
}

Received July 30, 2018; Accepted March 26, 2019

DOI: $10.3892 / \mathrm{ol} .2019 .10216$

\begin{abstract}
Application value of epidural anesthesia combined with epidural analgesia and general anesthesia combined with intravenous analgesia in ovarian cancer surgery was explored. In total 298 ASA I-III grade patients with ovarian cancer, undergoing extensive total hysterectomy and pelvic lymphotomy, were retrospectively analyzed. Patients were divided into two groups: the epidural anesthesia combined with epidural analgesia group (group A, 158 cases), and the general anesthesia combined with intravenous analgesia group (group B, 140 cases). The first exhaust time, incidence of adverse reactions, Aldrete score, and recovery were observed, and the visual analogue scale (VAS) scores during resting, exercise and cough at $24 \mathrm{~h}$ after surgery were recorded. Fasting venous blood $(2 \mathrm{ml})$ was drawn at the same time before anesthesia and at $24 \mathrm{~h}$ after anesthesia in both groups to determinate cortisol (COR) and C-reactive protein (CRP) levels. The first exhaust time and incidence of adverse reactions in group $\mathrm{A}$ were significantly lower than those in group $\mathrm{B}(\mathrm{P}<0.05)$. The Aldrete score and extubation time (ET) in group A were significantly higher than that in group B. Eye opening time (EOT), recovery orientation time (ROT) and post-anesthesia care unit (PACU) time in group A were significantly lower than those in group $\mathrm{B}(\mathrm{P}<0.05)$. The VAS scores in group A during resting, exercise and cough were lower than those in group $\mathrm{B}$ $(\mathrm{P}<0.05)$. Compared with before anesthesia, the levels of COR and CRP increased significantly in both groups at $24 \mathrm{~h}$ after anesthesia $(\mathrm{P}<0.05)$, while the level of COR and CRP in
\end{abstract}

Correspondence to: Dr Yanlong Liu, Department of Anesthesiology, Jiangxi Provincial People's Hospital, 152 Aiguo Road, Nanchang, Jiangxi 330006, P.R. China

E-mail: yva6bz@163.com; liuyanlong2655043@126.com

Key words: epidural anesthesia combined with epidural analgesia, general anesthesia combined with intravenous analgesia, ovarian cancer, prognosis group A was significantly lower than that in group B, at $24 \mathrm{~h}$ after surgery $(\mathrm{P}<0.05)$. Epidural anesthesia combined with epidural analgesia has better analgesic effect, higher safety, lower incidence of adverse reactions, and is beneficial to the recovery of patients with ovarian cancer after radical operation when compared with general anesthesia combined with intravenous analgesia.

\section{Introduction}

Ovarian cancer, an epithelial malignant tumor originating from the female ovary, is the leading cause of mortality among gynecological malignancies (1). Due to the special anatomical position of the ovary, the initial symptoms are not obvious, and symptoms, such as, abdominal swelling, pelvic pain and inappetence, are gradually developed in the late stage (2). Ovarian cancer is more likely to recur and metastasize to peritoneum, liver and lung, with a low 5-year survival rate and poor prognosis (3). If diagnosed and treated in the early stages ovarian cancer has a great chance of cure. However, as it is located in the deep pelvic cavity and its early symptoms are not obvious, most people are in the middle and late stage by the time of diagnosis (4). Although the treatment of ovarian cancer is improving with the progress of medical science and technology, the current treatment of ovarian cancer is still surgery plus chemotherapy (5). Local anesthesia is widely used in cancer patients during surgery. This can range from pain control to reduced use of opioids (6).

Epidural anesthesia, also called epidural space block anesthesia, refers to the injection of local anesthetics into the epidural cavity to block the spinal nerve root and paralyze the area controlled by the spinal nerve (7). Epidural anesthesia is often used in abdominal, pelvic and lower limb surgeries, and is mainly divided into high, middle and low epidural anesthesia. High epidural anesthesia is also used for postoperative analgesia, i.e., epidural analgesia (8). General anesthesia inhibits the central nervous system of patients and is induced by inhalation, intravenous drip and intramuscular injection of general anesthetics, resulting in a loss of general pain sensation and relaxation of the muscles of the whole body (9). When the concentration of the anesthetic in vivo decreases 
due to metabolism, the inhibitory reaction gradually disappears and the patient gradually returns to consciousness (10). Intravenous analgesia is one of the analgesic methods of systemic administration, referring to the effect of systemic analgesia by venous pump or intravenous drip of opioids and certain non-steroidal drugs. Since respiratory depression may occur, ECG monitoring is required for analgesia at the same time $(11,12)$. Inhalation anesthetics and intravenous opioids may inhibit cellular immunity by reducing the activity of natural killer cells. Axial nerve anesthesia (including epidural or spinal anesthesia) combined with general anesthesia can alleviate neuroendocrine stress response and prevent immunosuppression (13).

Ovarian cancer surgery can cause great trauma to patients' body function and immune function. In addition, a poor postoperative analgesic effect will also bring great mental pain to patients (14). Studies have shown that different anesthesia methods during surgery and different analgesia methods after surgery can affect the postoperative recovery and recurrence rate in patients with breast and prostate cancer (15). Therefore, the application value and prognostic effect of epidural anesthesia combined with epidural analgesia and general anesthesia combined with intravenous analgesia on the prognosis of ovarian cancer was studied, to improve the recovery after radical surgery of ovarian cancer, reduce the occurrence of complications, improve the prognosis and increase the survival rate of patients.

\section{Patients and methods}

Patient data. Clinical data of 298 patients, with a mean age of $43.56 \pm 7.24$ years, undergoing radical ovarian cancer surgery from December 2015 to June 2017 in Jiangxi Provincial People's Hospital (Nanchang, China), were retrospectively analyzed. The patients were divided into two groups: the epidural anesthesia combined with epidural analgesia group (group A, 158 cases), and the general anesthesia combined with intravenous analgesia group (group B, 140 cases). Inclusion criteria: all patients who met the diagnostic criteria of ovarian cancer and were confirmed by pathology; patients with newly developed ovarian cancer; undergoing radical surgery for the first time; undergoing extensive total hysterectomy combined with pelvic lymph node dissection; with the same postoperative chemotherapy regimen, paclitaxel combined with carboplatin (TP regimen). Exclusion criteria: patients in group A with contraindications to epidural analgesia, and patients in group $\mathrm{B}$ with contraindications to general anesthesia and intravenous analgesia; patients suffering from other serious underlying diseases, such as, heart, liver or kidney diseases. This study was approved by the Ethics Committee of Jiangxi Provincial People's Hospital. Signed written informed consents were obtained from the patients or the guardians.

Materials and reagents. Atropine (SFDA approval no. H32020166; Jiangsu Lianhuan Pharmaceutical Co., Ltd., Yangzhou, China); lidocaine (SFDA approval no. H20043560; Cisen Pharmaceutical Co., Ltd., Jining, China); amethocaine (SFDA approval no. H20084308; Chengdu Tiantaishan Pharmaceutical Co., Ltd., Qionglai, China); adrenalin (SFDA approval no. H11021929; CR Double-Crane Pharmaceutical
Co., Ltd., Beijing, China); propofol (SFDA approval no. J20080023; Fresenius Kabi AB, Bad Homburg, Germany); fentanyl (SFDA approval no. H42022076; Yichang Renfu Pharmaceutical Co., Ltd., Yichang, China); atracurium (SFDA approval no. H20060869; Jiangsu Hengrui Medicine Co., Ltd., Lianyungang, China); sevoflurane (SFDA approval no. H20040771; Jiangsu Hengrui Medicine Co., Ltd.); ropivacaine (SFDA approval no. H20070066; Yangtze River Pharmaceutical Group Co., Ltd., Taizhou, China); flurbiprofen axetil (SFDA approval no. H20041508; Beijing Tide Pharmaceutical Co., Ltd., Beijing, China); cortisol (COR) radioimmunoassay kit and C-reactive protein (CRP) immunotransmission kit (both from Shanghai Xinfan Biotechnology Co., Ltd., Shanghai, China).

Anesthetic and analgesic grouping. All 298 patients undergoing radical ovarian cancer surgery were divided into group A and B: group A, epidural anesthesia combined with epidural analgesia; group B, general anesthesia combined with intravenous analgesia (16).

Preparation for anesthesia: drinking water $6 \mathrm{~h}$ before surgery and eating $4 \mathrm{~h}$ before surgery were prohibited. Atropine $(0.3 \mathrm{mg})$ was injected intramuscularly $30 \mathrm{~min}$ before anesthesia. In group A, puncture and epidural catheterization were performed at L3-L4 lumbar intervertebral space and T10-T11 thoracic intervertebral space. The vital signs of patients, such as, heart rate, blood pressure and oxygen saturation were monitored by ECG monitor. Anesthesia: in group A, 1.5\% lidocaine $3 \mathrm{ml}$ and $0.16 \%$ amethocaine and adrenalin $(200,000: 1)$ mixture were given by epidural administration, and the anesthesia level was controlled below T5. In group B, $2 \mathrm{mg} / \mathrm{kg}$ propofol, $0.6 \mu \mathrm{g} / \mathrm{kg}$ fentanyl and $0.6 \mathrm{mg} / \mathrm{kg}$ atracurium were injected intravenously to induce anesthesia; 1.3 MAC sevoflurane was inhaled using facemask and 0.3-0.5 mg fentanyl was injected intravenously for maintenance of anesthesia. Postoperative analgesia: $30 \mathrm{~min}$ before the end of surgery, group A was given epidural injection of $0.25 \%$ ropivacaine $5 \mathrm{ml}$ for the first time, then $100 \mathrm{ml}$ of $0.15 \%$ ropivacaine and $0.6 \mu \mathrm{g} / \mathrm{kg}$ fentanyl were continuously administered for epidural analgesia at a rate of $2 \mathrm{ml} / \mathrm{h}$. At $30 \mathrm{~min}$ before the end of surgery, group B was given intravenous drip of $5 \mu \mathrm{g} / \mathrm{kg}$ fentanyl and $200 \mathrm{mg}$ flurbiprofen axetil for the first time, then $100 \mathrm{ml}$ of $1.0 \mu \mathrm{g} / \mathrm{kg}$ fentanyl and $50 \mathrm{mg}$ flurbiprofen were continuously conducted for intravenous analgesia at a rate of $2 \mathrm{ml} / \mathrm{h}$.

Comparison of improved Aldrete score and recovery. The following were recorded: eye opening time (EOT), the period of time from the end of the surgery to the opening of the eyes; recovery orientation time (ROT), the period of time from the end of the surgery to the recovery of orientation ability; extubation time (ET), the period of time from the end of the surgery to the extubation; post-anesthesia care unit (PACU) time, the time from the end of the surgery to the transfer out of the recovery room. Aldrete score was also used to evaluate the recovery of patients from five aspects: activity, respiration, blood pressure, consciousness and $\mathrm{SpO}_{2}$. Total score was 10 , and the higher score indicated a better recovery. If the score was $>9$, the tube could be removed, and no abnormality after 15 min indicated that the patient could be transferred out of the recovery room and sent back to ICU for observation. 
Table I. Comparison of general clinical data between the two groups.

\begin{tabular}{|c|c|c|c|c|}
\hline Clinical data & Group A $(\mathrm{n}=158)$ & Group B (n=140) & $\mathrm{t} / \chi^{2}$ test & P-value \\
\hline Age (years) & $42.87 \pm 6.94$ & $43.87 \pm 8.11$ & 0.26 & 1.14 \\
\hline $\operatorname{BMI}\left(\mathrm{kg} / \mathrm{m}^{2}\right)$ & $22.43 \pm 5.61$ & $23.54 \pm 6.43$ & 1.58 & 0.12 \\
\hline ASA classification & & & 0.00 & 0.99 \\
\hline I & $22(13.92)$ & $20(14.29)$ & & \\
\hline II & $110(69.62)$ & 97 (69.29) & & \\
\hline III & $26(16.46)$ & $23(16.43)$ & & \\
\hline TNM staging & & & 0.00 & 0.99 \\
\hline Stage I, II & $53(33.54)$ & $47(33.57)$ & & \\
\hline Stage III, IV & $105(66.46)$ & $93(66.43)$ & & \\
\hline Pathological classification & & & 0.73 & 0.12 \\
\hline Epithelial type & $86.00(54.43)$ & $79(56.43)$ & & \\
\hline Other & $72.00(45.57)$ & $61(43.57)$ & & \\
\hline Operative time (min) & $224.45 \pm 15.63$ & $225.32 \pm 17.43$ & 0.65 & 0.45 \\
\hline Bleeding volume (ml) & $484.24 \pm 164.24$ & $478.54 \pm 174.34$ & 0.29 & 0.77 \\
\hline Intestinal resection rate & $101(63.92)$ & $83(59.29)$ & 0.68 & 0.41 \\
\hline Diaphragmatic resection rate & $44(27.85)$ & $45(32.14)$ & 0.65 & 0.42 \\
\hline Splenectomy rate & $13(8.23)$ & $12(8.57)$ & 0.01 & 0.92 \\
\hline
\end{tabular}

Independent samples t-test and Chi-square test were used for comparison of the data between groups.

Visual analogue scale (VAS). VAS is widely used in clinical evaluation of pain. VAS scores during resting, exercise and cough at $24 \mathrm{~h}$ after surgery were recorded. The total score was 10 , and the higher the score, the higher the pain degree (painless, 0; severe pain, 10).

Stress response comparison. Fasting venous blood $(2 \mathrm{ml})$ was drawn at the same time before anesthesia and at $24 \mathrm{~h}$ after anesthesia in both groups to determinate COR and CRP levels. COR was detected by radioimmunoassay and CRP by immunotransmission turbidimetry. COR and CRP levels were compared between the two groups.

Other comparative indicators. The first exhaust time after surgery, the adverse reactions and the occurrence of complications were recorded. The adverse reactions after anesthesia included hypotension, pluritus, hypertension, nausea and vomiting, and lethargy.

Statistical analysis. SPSS 20.0 (IBM Corp., Armonk, NY, USA) was used for statistical analysis, and GraphPad Prism 7 (GraphPad Software, Inc., La Jolla, CA, USA) was used to draw the data illustrations. The Aldrete scores, the recovery, VAS scores, COR and CRP levels, and the first exhaust time after surgery were all expressed as mean \pm standard deviation (mean \pm SD) and analyzed by independent samples t-test. Paired t-test was used for the intragroup comparison of COR and CRP between before and after surgery. Independent samples t-test was used to compare the two groups at the same time-point. The incidence of adverse reactions and other enumeration data were expressed as $n(\%)$, and analyzed by $\chi^{2}$ test. Analysis of variance (ANOVA) was performed to assess the significance between multiple groups and least significant difference (LSD) test was used as a post hoc test. $\mathrm{P}<0.05$ was considered to indicate a statistically significant difference. Kaplan-Meier was used for survival analysis and log-rank test was used for comparison of the curves.

\section{Results}

Comparison of general clinical data between the two groups of patients. There was no significant difference in age, BMI, ASA classification, TNM staging, pathological classification, operative time, bleeding volume and resection rate of intestine, spleen and diaphragm between the two groups $(\mathrm{P}>0.05)$ (Table I).

Comparison of the first exhaust time and the incidence of adverse reactions after surgery between the two groups. The first exhaust time in group A was significantly lower than that in group $\mathrm{B}(\mathrm{P}<0.05)$ (Fig. 1). There were significant differences in hypotension, hypertension, pruritus, nausea and vomiting, and lethargy between the two groups (Table II).

Comparison of Aldrete scores and recovery between the two groups. The Aldrete score and ET in group A were significantly higher than that in group $\mathrm{B}(\mathrm{P}<0.05)$, and EOT, ROT and PACU time were significantly lower than that in group $\mathrm{B}$ $(\mathrm{P}<0.05)$ (Table III).

Comparison of pain VAS scores between the two groups. VAS scores in group A during resting, exercise and cough at 
Table II. Comparison of the first exhaust time and the incidence of adverse reactions after surgery between the two groups.

\begin{tabular}{|c|c|c|c|c|}
\hline Variables & Group A $(n=158)$ & Group B $(n=140)$ & $\mathrm{t} / \chi^{2}$ test & P-value \\
\hline First exhaust time (h) & $32.53 \pm 5.54$ & $37.51 \pm 6.46$ & 7.10 & $<0.001$ \\
\hline Adverse reactions incidence $(\%)$ & $16(10.13)$ & $28(20)$ & 5.75 & 0.02 \\
\hline Hypotension & $2(1.27)$ & $9(6.43)$ & 5.57 & 0.02 \\
\hline Pruritus & $3(1.90)$ & $13(9.29)$ & 7.08 & $<0.001$ \\
\hline Hypertension & $8(5.06)$ & $17(12.14)$ & 4.84 & 0.03 \\
\hline Nausea and vomiting & $2(1.27)$ & $8(5.71)$ & 4.53 & 0.03 \\
\hline Lethargy & $0(0.00)$ & $0(0.00)$ & & \\
\hline
\end{tabular}

Independent samples t-test was used to analyze the first exhaust time and Chi-square test was used to compare the incidence of adverse reactions. The first exhaust time and the incidence of adverse reactions in group A were significantly lower than those in group B (P<0.05).

Table III. Comparison of Aldrete scores and recovery.

\begin{tabular}{|c|c|c|c|c|c|}
\hline Groups & Aldrete scores & EOT (min) & ROT (min) & $\mathrm{ET}(\mathrm{min})$ & PACU (min) \\
\hline Group A & $7.72 \pm 2.32$ & $10.83 \pm 4.83$ & $15.49 \pm 3.24$ & $23.45 \pm 6.32$ & $45.65 \pm 8.76$ \\
\hline Group B & $5.12 \pm 1.43$ & $15.43 \pm 6.43$ & $22.43 \pm 5.43$ & $18.54 \pm 7.53$ & $49.76 \pm 9.65$ \\
\hline $\mathrm{t}$ test & 11.79 & 6.91 & 13.19 & 6.05 & 3.83 \\
\hline P-value & $<0.001$ & $<0.001$ & $<0.001$ & $<0.001$ & $<0.001$ \\
\hline
\end{tabular}

Independent samples t-test was used for analysis. EOT, eye opening time; ROT, recovery orientation time; ET, extubation time; PACU, post-anesthesia care unit.

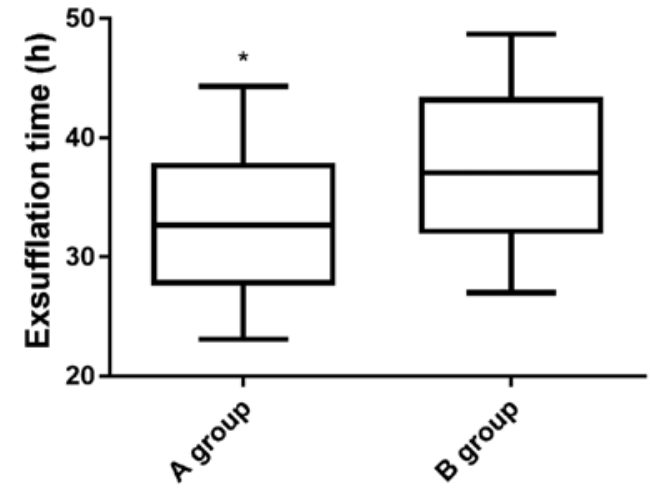

Figure 1. Comparison of the first exhaust time after operation between the two groups. Independent samples t-test was used to analyze the first exhaust time. The first exhaust time in group A was significantly lower than that in group $\mathrm{B}\left({ }^{*} \mathrm{P}<0.05\right)$.

$24 \mathrm{~h}$ after surgery were $1.83 \pm 0.58,2.23 \pm 0.67$ and $2.11 \pm 0.64$, respectively; and in group B were $2.290 \pm 0.63,2.98 \pm 0.77$ and $2.87 \pm 0.68$, respectively. The VAS scores in group A during resting, exercise and cough were lower than those in group B $(\mathrm{P}<0.05)$ (Fig. 2).

Comparison of stress response between the two groups. Before anesthesia: the level of COR in group A was $217.53 \pm 37.54 \mathrm{ng} / \mathrm{ml}$, and the level of CRP was $4.21 \pm 0.81 \mathrm{mg} / \mathrm{ml}$. The level of COR in group B was $221.43 \pm 43.21 \mathrm{ng} / \mathrm{ml}$, and the level of CRP was $4.36 \pm 1.01 \mathrm{mg} / \mathrm{ml}$. Twenty-four hours after

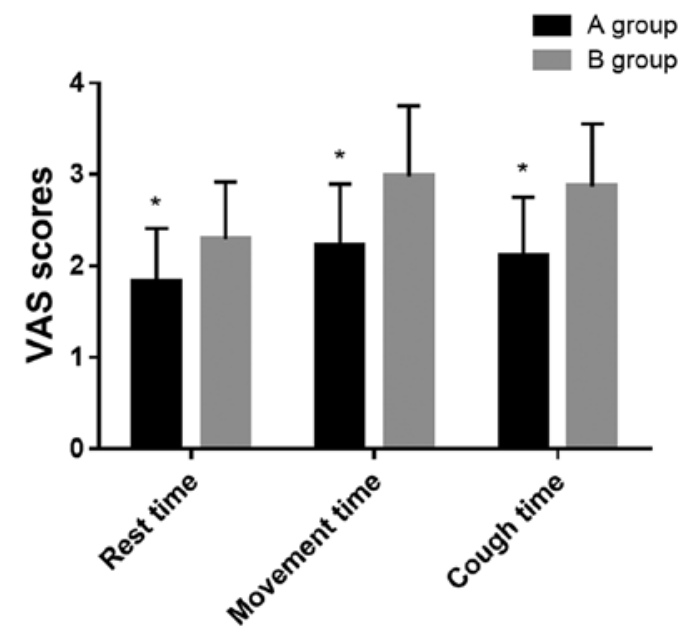

Figure 2. Comparison of VAS scores between the two groups after surgery. Independent samples t-test was used for statistical analysis. VAS scores in group A, during resting, exercise and cough, were lower than those in group $\mathrm{B}\left({ }^{*} \mathrm{P}<0.05\right)$. VAS, visual analogue scale.

surgery: the level of COR in group A was $415.34 \pm 48.76 \mathrm{ng} / \mathrm{ml}$, and the level of CRP was $32.54 \pm 5.43 \mathrm{mg} / \mathrm{ml}$. The level of COR in group B was $537.32 \pm 54.59 \mathrm{ng} / \mathrm{ml}$, and the level of CRP was $46.55 \pm 8.54 \mathrm{mg} / \mathrm{ml}$. There was no difference in the levels of COR and CRP between the two groups before anesthesia $(P>0.05)$. However, compared with before anesthesia, the levels of COR and CRP increased significantly in both groups at $24 \mathrm{~h}$ after surgery $(\mathrm{P}<0.05)$. Also, the COR and CRP levels 


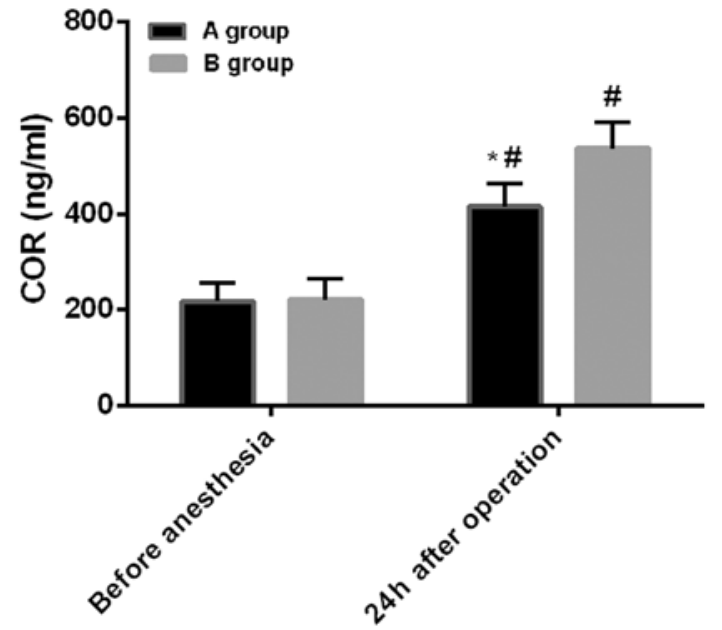

Figure 3. Comparison of COR levels between the two groups. The intragroup comparisons of COR before and after anesthesia were analyzed by paired $\mathrm{t}$-test, and the comparisons between the two groups at the same time-point were performed by independent samples t-test. There was no difference in the level of COR between the two groups before anesthesia ( $P>0.05)$. At $24 \mathrm{~h}$ after surgery, the levels of COR increased significantly in both groups, compared with before anesthesia $\left({ }^{*} \mathrm{P}<0.05\right)$. The level of $\mathrm{COR}$ in group A was significantly lower than that in group $\mathrm{B}$ at $24 \mathrm{~h}$ after surgery $\left({ }^{*} \mathrm{P}<0.05\right)$. COR, cortisol.

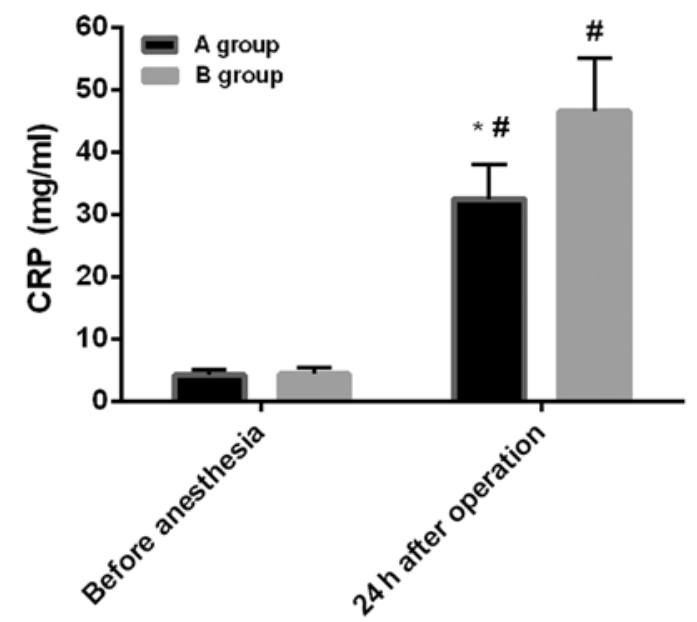

Figure 4. Comparison of CRP levels between the two groups. The intragroup comparisons of CRP before and after anesthesia were analyzed by paired t-test, and the comparisons between the two groups at the same time-point were performed by independent samples t-test. There was no difference in the level of CRP between the two groups before anesthesia $(\mathrm{P}>0.05)$. At $24 \mathrm{~h}$ after surgery, the levels of CRP increased significantly in both groups, compared with before anesthesia $\left({ }^{\#} \mathrm{P}<0.05\right)$. The level of CRP in group A was significantly lower than that in group $\mathrm{B}$ at $24 \mathrm{~h}$ after surgery $\left({ }^{*} \mathrm{P}<0.05\right)$. CRP, C-reactive protein.

in group A were significantly lower than those in group B at $24 \mathrm{~h}$ after surgery $(\mathrm{P}<0.05)$ (Figs. 3 and 4$)$.

One-year survival rate between the two groups. In group A (without any cases lost to follow-up), 7 patients died, 1 year after operation, and the survival rate was $95.57 \%$. In group B (without any cases lost to follow-up), 4 patients died, 1 year after operation, and the survival rate was $97.14 \%$. The survival rate of group A was lower than that of group B. Log-rank test showed that there was no significant difference in survival rate between groups A and B (P>0.05) (Fig. 5).

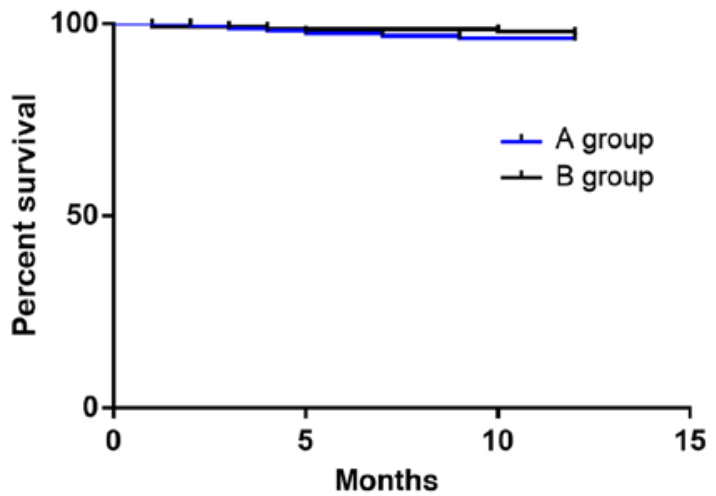

Figure 5. Survival curves of the two groups. Kaplan-Meier was performed for survival analysis. The survival rate of group A was $95.57 \%$, and that of group B was $97.14 \%$. The survival rate of group A was lower than that of group $\mathrm{B}$. There was no significant difference between the two groups $(\mathrm{P}>0.05)$.

\section{Discussion}

Ovarian cancer is one of the three major causes endangering the health of women. Because the specific molecular pathogenesis of ovarian cancer is still unclear and there is no effective early diagnosis method, $3 / 4$ patients are in the late stage at diagnosis. Ovarian cancer is easy to recur and metastasize, the overall curative effect is not high and the prognosis is poor $(17,18)$. Surgical resection is the main treatment of this disease. Studies have shown that perioperative factors, including anesthetic and analgesic techniques, may affect postoperative recovery (19). It has been estimated that there were 14.1 million new cancer cases worldwide in 2012, and the number is expected to increase to 21.7 million by 2030 . Although the proportion of patients undergoing subsequent surgical resection is $\sim 5-80 \%$ depending on the type of tumor, surgical resection is still the best chance of long-term survival for many solid cancers. However, paradoxically, there is evidence that surgery itself may be associated with the proliferation or metastasis of tumors. Surgical removal can destroy tumors and blood vessels supplying tumors, leading to the spread of tumor cells to the peripheral circulation (20). Therefore, in the present study, the application value and prognostic effect of epidural anesthesia combined with epidural analgesia and general anesthesia combined with intravenous analgesia on the prognosis of ovarian cancer was explored to improve the recovery after radical surgery of ovarian cancer, reduce the occurrence of complications, improve the prognosis and increase the survival rate of patients.

The first exhaust time and adverse reaction incidence in group A were significantly lower than those in group B, which indicated that compared with general anesthesia combined with intravenous analgesia, the recovery rate of gastrointestinal tract is faster and safer compared to epidural anesthesia combined with epidural analgesia. Panaretou et al (21) have studied the effects of epidural anesthesia and analgesia on gastrointestinal pressure in patients undergoing aortic reconstruction surgery, and have found that epidural anesthesia and analgesia could relieve gastrointestinal pressure, which is consistent with the results in our study. In this study, it was also found that the first exhaust time after epidural anesthesia combined with epidural analgesia was earlier, which indicated that the gastrointestinal 
tract recovered faster, so the gastrointestinal pressure was also smaller. Aldrete score and ET in group A were significantly higher than that in group B, and EOT, ROT and PACU time were significantly lower than that in group B. These results showed that compared with general anesthesia combined with intravenous analgesia, epidural anesthesia combined with epidural analgesia could significantly improve Aldrete scores and promote the recovery of patients. This may be due to the fact that epidural anesthesia combined with epidural analgesia is local anesthesia, while general anesthesia combined with intravenous analgesia is general anesthesia, and the EOT, ROT, ET and PACU time of general anesthesia is usually higher than that of local anesthesia (14). VAS scores in group A during resting, exercise and cough were lower than those in group B, which indicated that compared with general anesthesia combined with intravenous analgesia, epidural anesthesia combined with epidural analgesia could significantly reduce the degree of postoperative pain, and the analgesic effect is more obvious. Stress response is a non-specific self-defense response, such as, elevated blood sugar, increased blood pressure and faster heart rate caused by sudden stimuli like surgery, trauma, and hunger (22). COR is a glucocorticoid produced by renicapsule after stress stimulation (23), CRP is a typical acute phase protein and inflammatory protein (24), both of which can reflect the stress response of the body (25). Radical resection of ovarian cancer can cause great trauma and severe postoperative pain, and changes in the body's internal environment are likely to cause perioperative stress response (26). Compared with before anesthesia, the levels of COR and CRP increased significantly at $24 \mathrm{~h}$ after surgery in both groups, indicating that the surgery stimulated the body to undergo perioperative stress reactions. Sharp increase of COR and CRP, excessive inflammation, immune and stress response damage the body and are not conducive to postoperative recovery. The levels of COR and CRP in group A were significantly lower than that in group B at $24 \mathrm{~h}$ after surgery, confirming that compared with general anesthesia combined with intravenous analgesia, the epidural anesthesia combined with epidural analgesia could improve the perioperative stress response and reduce the degree of inflammatory reaction. Finally, we analyzed the 1-year survival rate between the two groups, and the results showed that there was no difference in the survival rate between the two groups. Christopherson et al (27) have found that epidural anesthesia could improve the survival rate of patients with non-metastatic colon cancer for 1.46 years, but has no effect on the survival of patients with metastatic colon cancer.

The present study found that the analgesic effect of epidural anesthesia combined with epidural analgesia is better than that of general anesthesia combined with intravenous analgesia. Moslemi et al (28) have found that with simple epidural analgesia in gynecological tumor surgery it is difficult to control intractable pelvic nerve pain, while combining intravenous analgesia with fentanyl, ketamine, lidocaine and analgesics a better analgesic effect can be achieved without any significant complications. This suggests that optimizing the combination of epidural anesthesia and epidural analgesia, i.e., combining with intravenous analgesia, can improve the analgesic effect when controlling certain intractable pain. Han et al (29) have found that compared with intravenous anesthesia alone, epidural anesthesia combined with intravenous anesthesia has less adverse effects on immune function in patients with ovarian cancer. Therefore, it is speculated that epidural analgesia combined with intravenous analgesia may have better analgesic effect and safety, which need to be verified in subsequent experiments. Also, the optimal dosage and order of use between the two groups needs further evaluation.

In this study, the efficacy of two groups of anesthesia was evaluated by comparing the first exhaust time, the incidence of adverse reactions, Aldrete score, recovery and related factors. However, there are still some limitations in this study. For example, no in-depth study of the regulation mechanism of anesthesia in patients was conducted.

In conclusion, compared with general anesthesia and intravenous analgesia, epidural anesthesia combined with epidural analgesia has better analgesic effect, higher safety and lower incidence of adverse reactions, and it is beneficial to the recovery of patients with ovarian cancer after radical operation.

\section{Acknowledgements}

Not applicable.

\section{Funding}

No funding was received.

\section{Availability of data and materials}

The datasets used and/or analyzed during the present study are available from the corresponding author on reasonable request.

\section{Authors' contributions}

SZ drafted the manuscript. SZ and XiaoxueZ were mainly devoted to collecting and interpreting the data. XiaomeiZ and YL assisted with VAS scores. All authors read and approved the final manuscript.

\section{Ethics approval and consent to participate}

The study was approved by the Ethics Committee of Jiangxi Provincial People's Hospital (Nanchang, China). Signed written informed consents were obtained from the patients or the guardians.

\section{Patient consent for publication}

Not applicable.

\section{Competing interests}

The authors declare that they have no competing interests.

\section{References}

1. Jacobs IJ, Menon U, Ryan A, Gentry-Maharaj A, Burnell M, Kalsi JK, Amso NN, Apostolidou S, Benjamin E, Cruickshank D, et al: Ovarian cancer screening and mortality in the UK Collaborative Trial of Ovarian Cancer Screening (UKCTOCS): A randomised controlled trial. Lancet 387: 945-956, 2016. 
2. Ginestra A, Miceli D, Dolo V, Romano FM and Vittorelli ML Membrane vesicles in ovarian cancer fluids: A new potential marker. Anticancer Res 19: 3439-3445, 1999.

3. Yuan X, Zhang J, Li D, Mao Y, Mo F, Du W and Ma X: Prognostic significance of tumor-associated macrophages in ovarian cancer: A meta-analysis. Gynecol Oncol 147: 181-187, 2017.

4. Zhang H, Liu T, Zhang Z, Payne SH, Zhang B, McDermott JE, Zhou JY, Petyuk VA, Chen L, Ray D, et al; CPTAC Investigators: Integrated proteogenomic characterization of human high-grade serous ovarian cancer. Cell 166: 755-765, 2016.

5. Zhang W, Liu R, Tang C, Xi Q, Lu S, Chen W, Zhu L, Cheng J, Chen Y, Wang W, et al: PFTK1 regulates cell proliferation, migration and invasion in epithelial ovarian cancer. Int $\mathbf{J}$ Biol Macromol 85: 405-416, 2016.

6. Xuan W, Zhao H, Hankin J, Chen L, Yao S and Ma D: Local anesthetic bupivacaine induced ovarian and prostate cancer apoptotic cell death and underlying mechanisms in vitro. Sci Rep 6: 26277, 2016.

7. Gottschalk A and Poepping DM: Epidural analgesia in combination with general anesthesia. Anasthesiol Intensivmed Notfallmed Schmerzther 50: 484-493, quiz 494-495, 2015 (In German).

8. Xing CY, Wu MY and Fan HP: Effects of different anesthetic and analgesic protocols on cellular immune function and stress hormone level in patients undergoing lobectomy for esophagus cancer. Nan Fang Yi Ke Da Xue Xue Bao 30: 284-287, 2010 (In Chinese)

9. Davidson AJ, Disma N, de Graaff JC, Withington DE, Dorris L, Bell G, Stargatt R, Bellinger DC, Schuster T, Arnup SJ, et al; GAS consortium: Neurodevelopmental outcome at 2 years of age after general anaesthesia and awake-regional anaesthesia in infancy (GAS): An international multicentre, randomised controlled trial. Lancet 387: 239-250, 2016.

10. Sun LS, Li G, Miller TL, Salorio C, Byrne MW, Bellinger DC, Ing C, Park R, Radcliffe J, Hays SR, et al: Association between a single general anesthesia exposure before age 36 months and neurocognitive outcomes in later childhood. JAMA 315: 2312-2320, 2016

11. Green SM, Rothrock SG, Harris T, Hopkins GA, Garrett W and Sherwin T: Intravenous ketamine for pediatric sedation in the emergency department: Safety profile with 156 cases. Acad Emerg Med 5: 971-976, 1998.

12. Nie Y, Liu Y, Luo Q and Huang S: Effect of dexmedetomidine combined with sufentanil for post-caesarean section intravenous analgesia: A randomised, placebo-controlled study. Eur J Anaesthesiol 31: 197-203, 2014.

13. Weng M, Chen W, Hou W, Li L, Ding M and Miao C: The effect of neuraxial anesthesia on cancer recurrence and survival after cancer surgery: An updated meta-analysis. Oncotarget 7: $15262-15273,2016$.

14. Li S: Effect of different surgical treatment on immune function of patients with benign ovarian tumor. J Clin Med Practice 21: 93-96, 2017 (In Chinese).

15. Lindholm ML, Granath F, Eriksson LI and Sandin R: Malignant disease within 5 years after surgery in relation to duration of sevoflurane anesthesia and time with bispectral index under 45 Anesth Analg 113: 778-783, 2011.

16. Garnett RL, MacIntyre A, Lindsay P, Barber GG, Cole CW, Hajjar G, McPhail NV, Ruddy TD, Stark R and Boisvert D: Perioperative ischaemia in aortic surgery: Combined epidural/general anaesthesia and epidural analgesia vs general anaesthesia and i.v. analgesia. Can J Anaesth 43: 769-777, 1996.
17. Claus EB, Schildkraut JM, Thompson WD and Risch NJ: The genetic attributable risk of breast and ovarian cancer. Cancer 77: 2318-2324, 1996.

18. Yhim HY, Jang MJ, Bang SM, Kim KH, Kim YK, Nam SH, Bae SH, Kim SH, Mun YC, Kim I, et al: Incidence of venous thromboembolism following major surgery in Korea: From the Health Insurance Review and Assessment Service database. J Thromb Haemost 12: 1035-1043, 2014.

19. Parat MO (ed): Morphine and metastasis: From bench to bedside. In: Morphine and Metastasis. Springer, Dordrecht, pp1-13, 2013

20. Iwasaki M, Zhao H, Jaffer T, Unwith S, Benzonana L, Lian Q, Sakamoto A and Ma D: Volatile anaesthetics enhance the metastasis related cellular sign alling including CXCR2 of ovarian cancer cells. Oncotarget 7: 26042-26056, 2016.

21. Panaretou V, Siafaka I, Theodorou D, Manouras A, Seretis C, Gourgiotis S, Katsaragakis S, Sigala F, Zografos G and Filis K: Combined general-epidural anesthesia with continuous postoperative epidural analgesia preserves sigmoid colon perfusion in elective infrarenal aortic aneurysm repair. Saudi J Anaesth 6: 373-379, 2012.

22. Rom O and Reznick AZ: The stress reaction: A historical perspective. Adv Exp Med Biol 905: 1-4, 2016.

23. Billing AM, Fack F, Renaut J, Olinger CM, Schote AB, Turner JD and Muller CP: Proteomic analysis of the cortisol-mediated stress response in THP-1 monocytes using DIGE technology. J Mass Spectrom 42: 1433-1444, 2007.

24. Thom SR: Oxidative stress is fundamental to hyperbaric oxygen therapy. J Appl Physiol (1985) 106: 988-995, 2009.

25. Shamsdin SA, Anvar M and Mehrabani D: The effect of exam stress on serum IL-6, cortisol, CRP and IgE levels. Iran Red Crescent Med J 12: 484-488, 2010

26. Sendasgupta C, Makhija N, Kiran U, Choudhary SK, Lakshmy R and Das SN: Caudal epidural sufentanil and bupivacaine decreases stress response in paediatric cardiac surgery. Ann Card Anaesth 12: 27-33, 2009.

27. Christopherson R, James KE, Tableman M, Marshall $\mathrm{P}$ and Johnson FE: Long-term survival after colon cancer surgery: A variation associated with choice of anesthesia. Anesth Analg 107: 325-332, 2008

28. Moslemi F, Rasooli S, Baybordi A and Golzari SE: A comparison of patient controlled epidural analgesia with intravenous patient controlled analgesia for postoperative pain management after major gynecologic oncologic surgeries: A randomized controlled clinical trial. Anesth Pain Med 5: e29540, 2015.

29. Han XR, Wen X, Li YY, Fan SH, Zhang ZF, Li H, Sun XF, Geng GQ, Sun S, Huang SQ, et al: Effect of different anesthetic methods on cellular immune functioning and the prognosis of patients with ovarian cancer undergoing oophorectomy. Biosci Rep 37: pii: BSR20170915, 2017.

This work is licensed under a Creative Commons Attribution-NonCommercial-NoDerivatives 4.0 International (CC BY-NC-ND 4.0) License. 